\title{
The Influence of Age on Some Ocular Basement Membranes
}

\author{
R. F. FISHER \\ London
}

\begin{abstract}
Summary
(1) Changes with age in the Young's modulus of elasticity of human lens capsule and Bruch's membrane have been determined. In lens capsule the modulus decreases with age whereas in Bruch's membrane it increases.

(2) Changes with age in the hydraulic conductivity of the human lens capsule and Bruch's membrane have also been determined and again show opposite changes with age. In the former membrane the hydraulic conductivity increases while in the latter it decreases.

(3) The ageing changes in the respective membranes indicate that, in man, some time after 100 years in all cases the lens capsule would have a similar hydraulic conductivity to lenses with advanced cataract, while in the same subject Bruch's membrane would have lost its permeability to water.
\end{abstract}

In the last century, those tissues which were observed by light microscopy and which appeared to be structureless were named 'glass membranes'. In the eye these are Decemet's membrane, lens capsule and Bruch's membrane. The arrangement of tissues adjacent to lens capsule or Bruch's membrane are similar (Fig. 1). The arrangement comprises a nutrient surface, aqueous or chorio-capillaris associated with a complex tissue of lens fibres, or rods and cones. Interdeposed between surface and tissue is a 'glass membrane' in contact with an epithelium from which it is thought to be derived. Electron-microscopy however has revealed that these apparently homogeneous glass membranes have a structure peculiar to the membrane concerned. In the case of lens capsule the membrane is a homogeneous matrix of layered filaments ${ }^{1}$ (Fig. 2a), whereas Bruch's membrane has a much more complex structure $^{2}$ (Fig. 2b).

The structural difference between lens cap- sule and Decemet's membrane is not very great and their respective permeabilities are similar, so in this paper only the lens capsule and Bruch's membrane are considered. Hitherto changes with age in Bruch's membrane have been confined to the electron-microscopic appearances of the membrane and its associated retinal pigment cells. ${ }^{3,4}$ In the present paper two biophysical properties of this membrane and their changes with age have been investigated, namely elasticity and hydraulic permeability, and contrasted with lens capsule for which these properties have already been reported. . $^{5.6}$

\section{Method}

The choroid was dissected out from 12 human eyes ranging from 20 to 80 years old and a piece of choroid from the posterior pole was carefully brushed on its retinal surface to remove the pigment cells. The chorio-capillaris was subsequently removed by means of a scalpel blade, the resulting almost transparent membrane being pared down to 


\section{GLASS MEMBRANES}

LENS
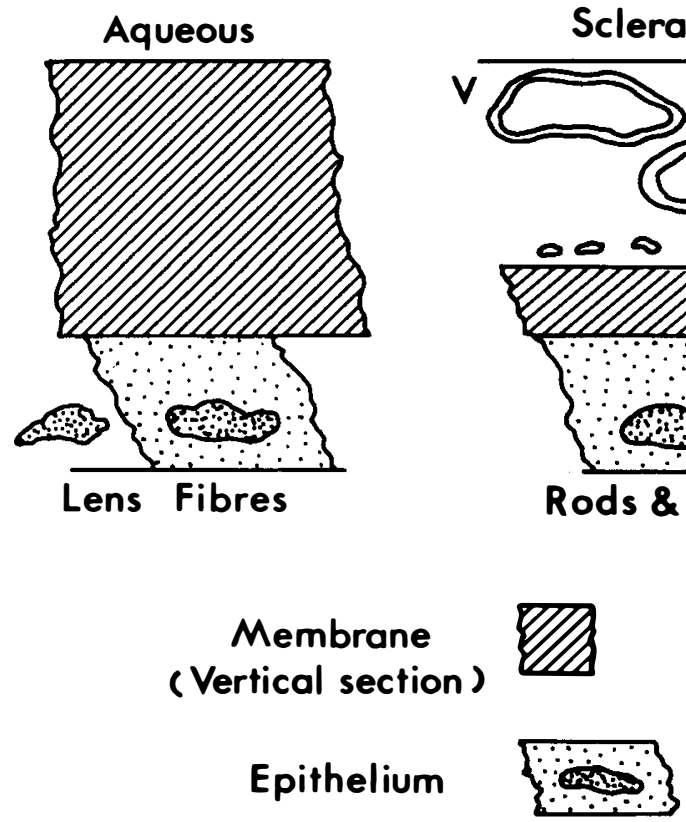

RETINA

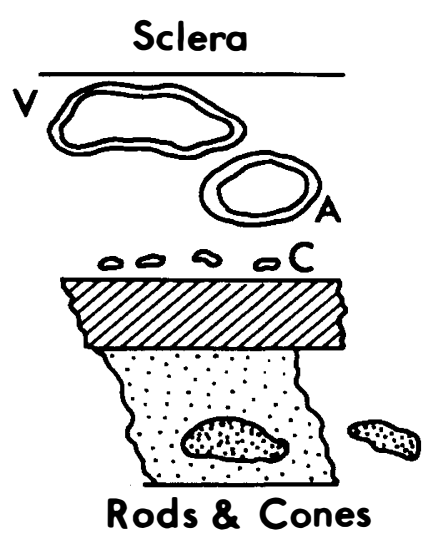

CORNEA

Aqueous

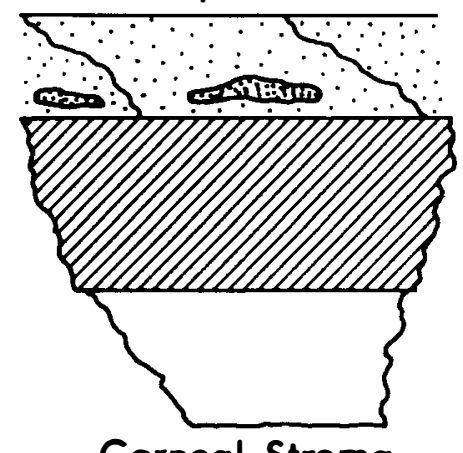

Corneal Stroma

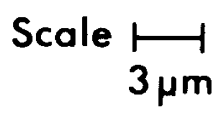

Fig. 1. Glass membranes of the eye in relation to other tissues.

\section{LENS}

\section{RETINA}
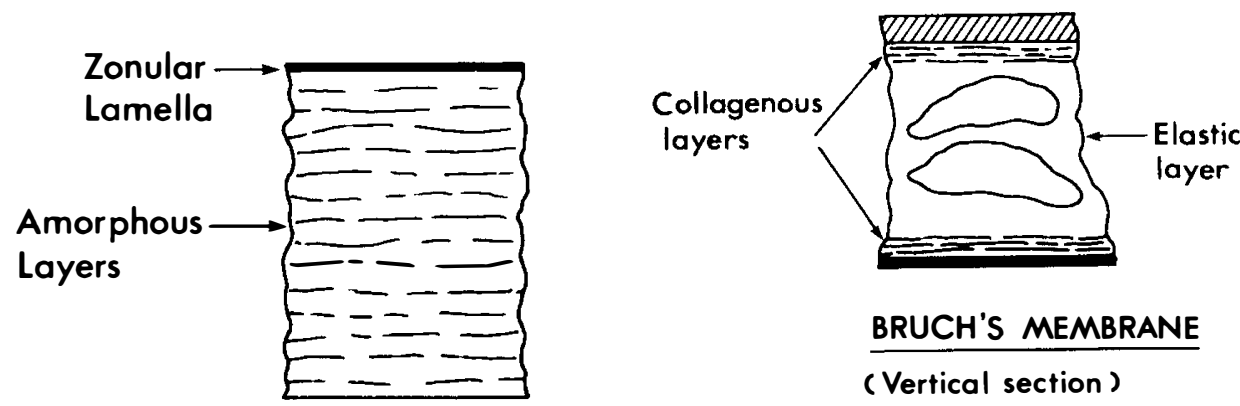

\section{BRUCH'S MEMBRANE \\ (Vertical section)}

LENS CAPSULE

\section{(Vertical section)}

\section{Scale}

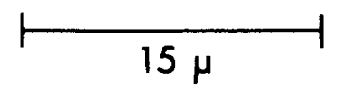

(a)

(b)

Basement membrane of

Pigment Epithelium

Choriocapillaris

Scale $3 \mu \mathrm{m}$

Fig. 2. Comparison of the structure of (a) lens capsule and (b) Bruch's membrane. 

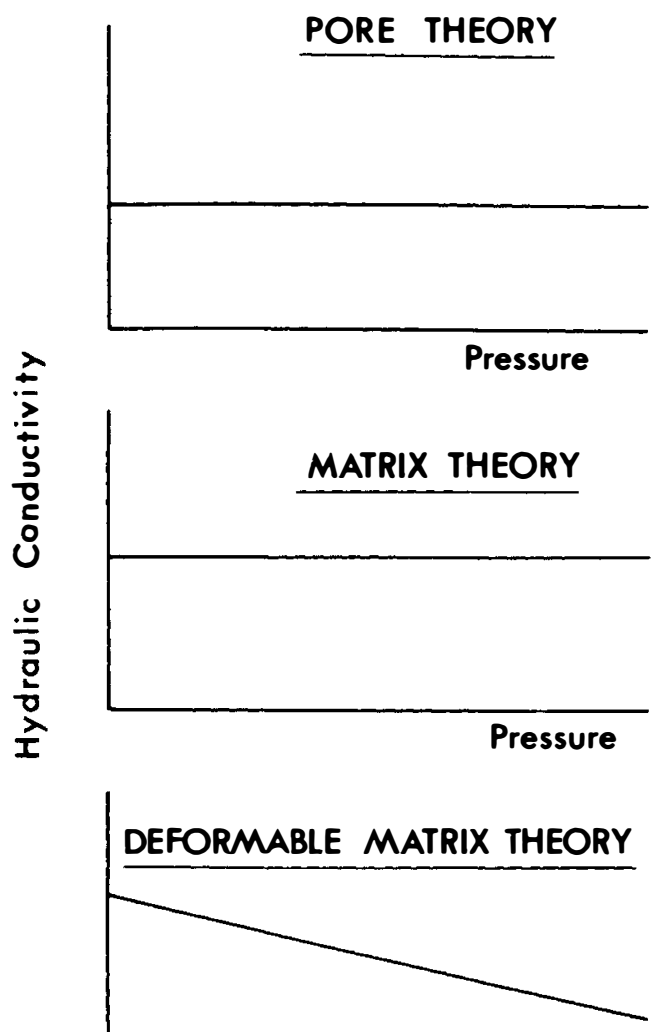

Fig. 3. Comparison of theories of permeability of glass membranes to pressure

Note: Both the basement membrane of the lens (lens capsule and Bruch's membrane show a decrease in hydraulic conductivity with pressure as predicted by the deformable matrix theory.

a thickness of about 5 to 6 microns. The membrane was then clamped between 2 stainless steel circular plates and drilled with a central hole $(2.5 \mathrm{~mm}$ diameter). Hydraulic permeability was determined at varying pressures up to a maximum of $2,700 \mathrm{~Pa}$ by a previously described method ${ }^{6}$. Subsequently on the same specimen Young's modulus of elasticity was also determined by a previously described method?

In the past it has been thought that basement membrane consisted of pores or collagenous rods through or over which water percolated. Fisher ${ }^{6}$ showed that the membrane of lens capsule deformed and decreased in thickness with the pressure necessary to cause water to pass through it. In consequence the hydraulic conductivity was not constant and independent of the applied pressure. In fact, it decreased with pressure and a deformable matrix theory was developed to explain this (Fig. 3). As Bruch's membrane behaves in a similar manner, for the purpose of the comparison of permeability therefore of lens capsule and Bruch's membrane it is important that each is measured at a similar stress and therefore deformation throughout the age range. Since lens capsule on average is about 5 times the thickness of Bruch's membrane, to obtain a similar degree of stress in each membrane the hydraulic permeability of lens capsule was measured at 5 times the pressure of Bruch's membrane, namely $100 \mathrm{~mm}$ mercury and $20 \mathrm{~mm}$ mercury respectively.

\section{Results}

(a) Hydraulic conductivity

In Figure 4 the changes in hydraulic conductivity with age for lens capsule and Bruch's membrane respectively are shown. It will be seen that at the age of 40 years the hydraulic conductivity of lens capsule is about 5 times greater than Bruch's membrane. This could be expected since Bruch's membrane is about 5 times thinner than lens capsule. However, lens capsule and Bruch's membrane behave quite differently as the eye ages. For lens capsule the hydraulic conductivity increases with age whereas for Bruch's membrane the hydraulic conductivity decreases with age.

\section{(b) Young's modulus of elasticity}

In Figure 5 a comparison between Young's modulus of elasticity of lens capsule, Bruch's membrane and skeletal tissue is shown. As Bruch's membrane has a central core of elastic tissue it might be expected that its Young's modulus would be lower than lens capsule which is a modified form of collagen. However, as age advances the fibrous collagenous elements in Bruch's membrane increases, ${ }^{8}$ and by the age of 40 years the Young's modulus of elasticity of both glass membranes 'since Young's modulus of lens capsule decreases with age' 5 are about equal.

\section{Discussion}

The hydraulic permeability of these glass membranes has an important bearing on the well being of the complex tissues which they separate from their nutrient source (see Fig. 1), so the respective values of each membrane of hydraulic conductivity are compared with capillary walls (Fig. 6). It will be seen 


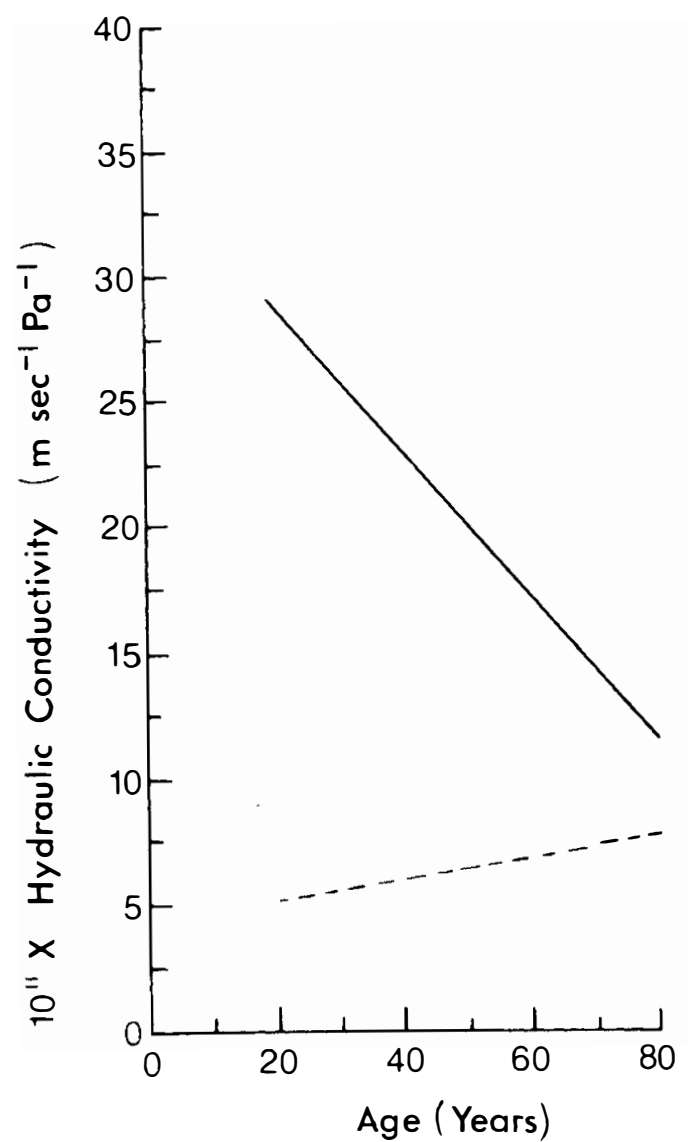

Fig. 4. A comparison of the hydraulic conductivity of lens capsule and Bruch's membrane and the changes which occur with age

Notes:

(a) Lens Capsule shown ----

Ages 4 to 69 years number of capsules $=25$. Hydraulic conductivity measure at $100 \mathrm{~mm} / \mathrm{Hg}$. Best fitting linear regression for hydraulic conductivity and age

$\left\llcorner\mathrm{p}=40.9 \times 10^{-12}+0.373 \times 10^{-12} \mathrm{~A}\right.$ where

$\mathrm{A}=$ Age (years) $\llcorner\mathrm{p}=$ Hydraulic Conductivity $\mathrm{r}=0.679 \mathrm{p}<0.001$.

(b) Bruch's membrane shown

Ages 22-71 years number of membranes $=6$.

Hydraulic conductivity measured at $20 \mathrm{~mm} / \mathrm{Hg}$.

Best fitting linear regression for hydraulic conductivity and age

$L \mathrm{p}=337 \times 10^{-12}-2.6 \times 10^{-12} \mathrm{~A}$ where

$\mathrm{A}=$ Age (years) $L \mathrm{p}=$ Hydraulic Conductivity $\mathrm{r}=0.885 \mathrm{p}<0.02$.

that Bruch's membrane has a similar hydraulic conductivity to the glomerulus of the kidney and much above the permeability of cardiac capillaries. On the other hand lens capsule has a greater permeability than the latter but about the same as mesenteric capillaries (not shown). Neither membrane therefore offer negligible resistance to water transfer as was previously thought. ${ }^{9}$

The relationship between ageing changes and disease has always been a matter of great interest. So far as the eye is concerned two of the chief diseases associated with old age are cataract and macular degeneration, ${ }^{10}$ and the possible role of ageing permeability changes in lens capsule and Bruch's membrane will now be discussed briefly.

\section{(a) Age changes in the hydraulic} conductivity of lens capsule

A study of the various types of senile cataract in relation to the moisture content of the lens substance and the permeability of its capsule have been described. ${ }^{11}$ Figures 7 (a) and 7(b) show the relationship between cuneiform opacities and cuneiform opacities with water clefts. So far as cuneiform opacities are concerned there was no difference in the hydraulic conductivity of the capsules in

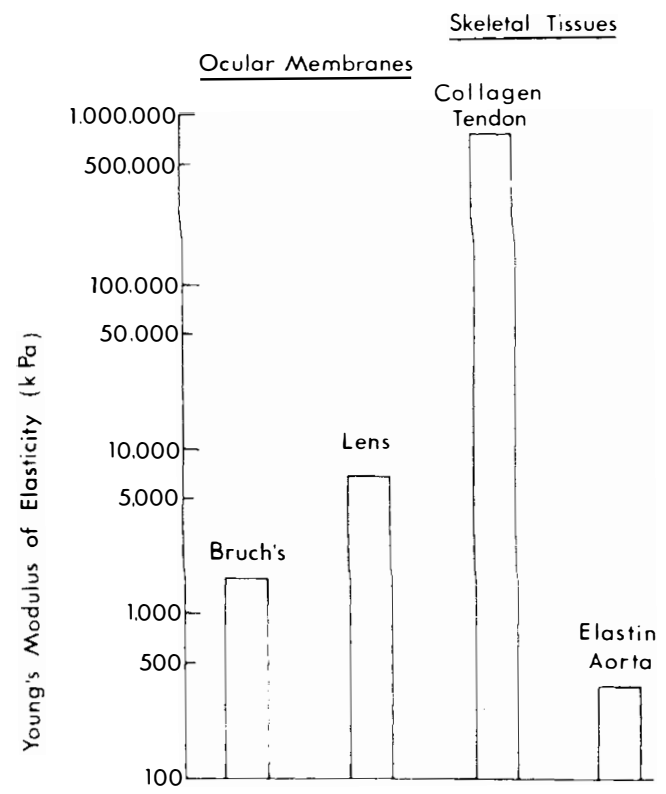

Fig. 5. The Young's modulus of elasticity of Bruch's membrane and lens capsule compared with the body connective tissues

Note: Young's modulus of elasticity about the age of 20 years for both lens capsule and Bruch's membrane. 


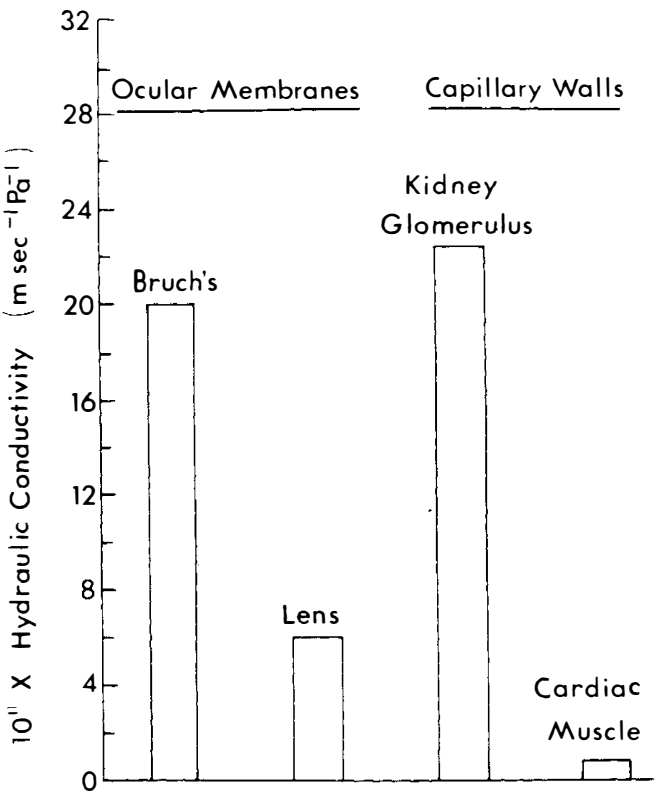

Fig. 6. The Hydraulic conductivity of Bruch's membrane and lens capsule compared with the walls of capillaries

Note: The hydraulic conductivity at about the age of 50 years for both lens capsule and Bruch's membrane.

lenses with cuneiform opacities (mean age 70) than the capsules of normal lenses (mean age $71)$. However, if in addition the lenses possessed water clefts in the cortex (mean age 74) the difference was significant, $\mathrm{p}<0.05$. The linear regression of increase in hydraulic conductivity with age predicts (Fig. 4) that all lenses will have gross water clefts and become intumescent by reason of the high water content of the cortex by about the age of 160 years.

(b) Age changes in hydraulic conductivity of Bruch's membrane

It has been suggested ${ }^{4}$ that Bruch's membrane might have reduced permeability to nutrients from the choroid because with increasing age there is a dramatic increase in the number of phagosomes and pigment granules within the membrane. This suggestion is now confirmed since the hydraulic conductivity of the membrane decreases dramatically with age (Fig. 4). Indeed, the linear regression of hydraulic conductivity with age for Bruch's membrane would indicate that no flow of water through the membrane should occur by the age of 130 years. It is reasonable therefore to postulate that the common occurrence of senile macular degeneration after the age of 70 years may be due in part at least, to the reduction in nutrient transfer between the chorio-capillaris and the retinal pigment cell as age advances.

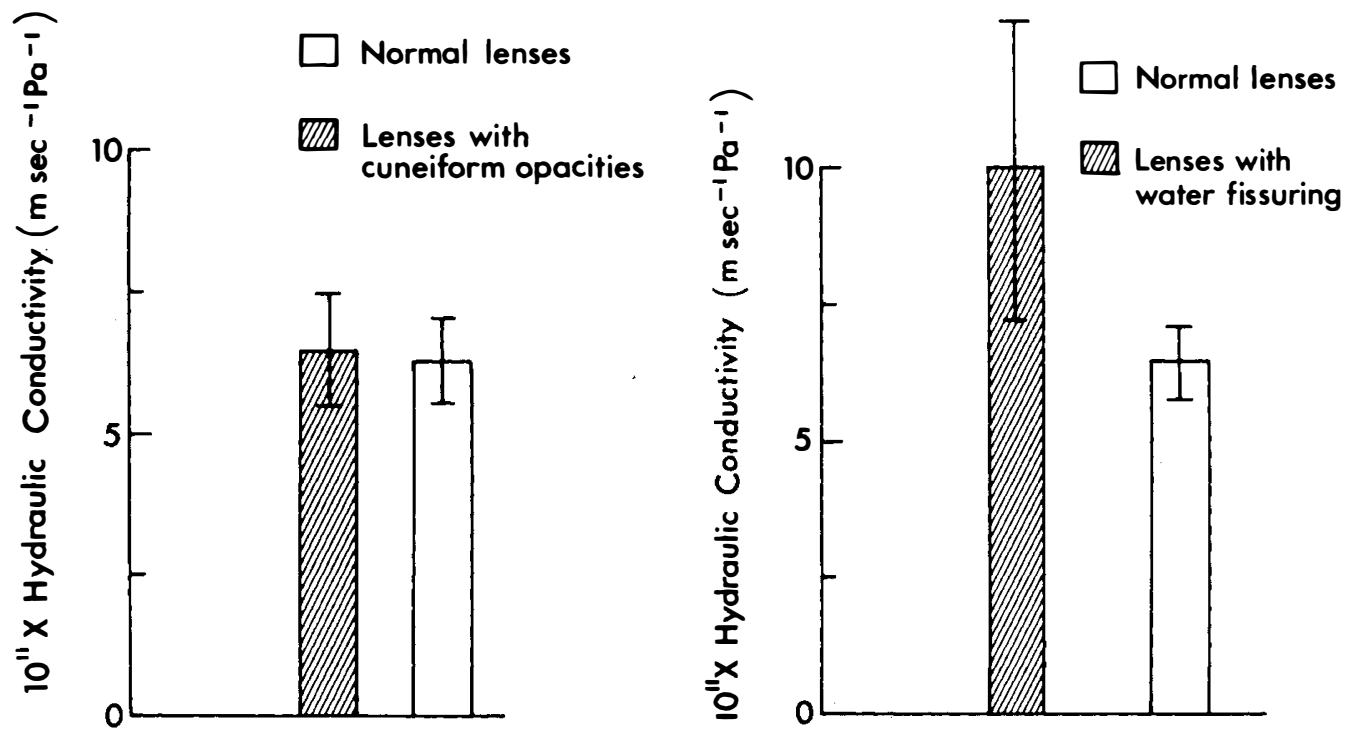

Fig. 7. Comparison of the hydraulic conductivity of the lens capsule in the presence of (a) cuneiform opacities and (b) cuneiform opacities with water fissuring 


\section{References}

${ }^{1}$ Fisher RF, Wakely J: The elastic constants and ultrastructural organization of basement membrane (lens capsule). Proc. R. Soc. B. 1976, 193: 33558.

2 Sumita R: Electron microscopic study of the choroid the fine structure of Bruch's membrane (lamina vitrea) in the choroid. Acta. Soc. Ophthalmol. Jap. 1961, 65: 1188-203.

${ }^{3}$ Kornzweig AL: Changes in choriocapillaris associated with senile macular degeneration. Ann. Ophthalmol. 1977, 9: 753-64.

${ }^{4}$ Grindle CFJ, Marshall J: Ageing changes in Bruch's membrane and their functional implications. Trans. Ophthalmol. Soc. UK 1978, 98: 172-5.

${ }^{5}$ Fisher RF: Elastic constants of the human lens capsule. J. Physiol. 1969, 201: 1-19.

${ }^{6}$ Fisher RF: The water permeability of basement membrane under increasing pressure: evidence for a new theory of permeability. Proc. R. Soc. B. 1982, 216: 475-96.

${ }^{7}$ Fisher RF, Hayes BP: Thickness and volume constants and ultrastructural organization of basement membrane (lens capsule). J. Physiol. 1979, 293: 229-45.

${ }^{8}$ Feher J, Valu L: Ageing changes of collagenous fibres of the choroid; investigation by polorization optics. Graefes. Arch. Klin. and Exper. Ophthalmol. 1967, 172: 249-53.

${ }^{9}$ Zurin KM, Henkind JVB: The retinal pigment epithelium. Chapter 1, pp 11-13. Cambridge Massachusetts Harvard University Press, 1979.

${ }^{10}$ Fisher RF: Eye disease and the ageing population (Treacher Collins Essay Prize). Trans. Ophthalmol. Soc. UK 1971, 91, 415-34.

${ }^{11}$ Fisher RF: Changes in the permeability of the lens capsule in senile cataract. Trans. Ophthalmol. Soc. UK 1977, 97, 50-103. 